\title{
1914-1918 ou l'expérience irréversible de Curzio Malaparte
}

1914-1918 o l'esperienza irreversibile di Curzio Malaparte

1914-1918, or Malaparte's Irreversible Experience

\section{Stéphanie Laporte}

\section{OpenEdition}

\section{Journals}

Édition électronique

URL : http://journals.openedition.org/cei/3278

DOI : 10.4000/cei.3278

ISSN : 2260-779X

\section{Éditeur}

UGA Éditions/Université Grenoble Alpes

Édition imprimée

ISBN : 978-2-84310-370-4

ISSN : 1770-9571

\section{Référence électronique}

Stéphanie Laporte, «1914-1918 ou l'expérience irréversible de Curzio Malaparte», Cahiers d'études italiennes [En ligne], 24 | 2017, mis en ligne le 28 février 2017, consulté le 27 mars 2021. URL : http:// journals.openedition.org/cei/3278; DOI : https://doi.org/10.4000/cei.3278

Ce document a été généré automatiquement le 27 mars 2021.

(c) ELLUG 


\title{
1914-1918 ou l'expérience irréversible de Curzio Malaparte
}

\author{
1914-1918 o l'esperienza irreversibile di Curzio Malaparte \\ 1914-1918, or Malaparte's Irreversible Experience
}

Stéphanie Laporte

\section{Peut-on lire Viva Caporetto! aujourd'hui ?}

1 «Non tutti potranno leggere questo libro »: dès les premiers mots, Viva Caporetto! résiste à ses lecteurs. Objet insolite et paradoxal, la singularité du premier livre de Curzio Malaparte se renforce sitôt qu'on a cru le définir. Singulier par son propos: transformer l'épisode militaire le plus honteux de l'histoire nationale en symbole de revanche, de rédemption et de renaissance pour les soldats meurtris du Karst; singulier par son auteur, héros de guerre qui s'auto-proclame caporettista sans jamais parler de ses médailles; singulier par ses choix narratifs, autobiographie authentique où le « je " s'efface derrière la foule anonyme des compagnons de tranchées; singulier par son vocabulaire politique, Caporetto y est décrit tour à tour comme expérience révolutionnaire du prolétariat et révélation mystique ; singulier au sein de la littérature de guerre, c'est un document historique, d'une actualité brûlante, qui appuie son authenticité sur une irréductible dimension allégorique; singulier par son parcours éditorial, il s'inscrit à la fois dans un contexte pacifiste d'après-guerre et dans celui du fascisme naissant. Aujourd'hui encore ce petit livre résiste avec force et n'a pas fini de questionner ses contemporains. Car peut-on lire Viva caporetto! aujourd'hui plus qu'hier? Peut-on le lire aujourd'hui mieux qu'hier?

2 Objet secret, livre ouvert à bien des méprises et malentendus, la diffusion du texte, qui a circulé et circule aujourd'hui encore dans ses deux versions - celle de 1921 dans les éditions de Isnenghi et de Biondi, celle de 1923 dans les éditions de Falqui et de Martellini - continue près de cent ans après sa parution d'ouvrir de mauvaises pistes ${ }^{1}$. Or comme le dit justement Marino Biondi dans une étude très détaillée sur le sujet ${ }^{2}$, il importe de revenir à la source du texte, à la première version de 1921 (publié sous le 
titre Viva Caporetto! en février, puis à nouveau en avril, sans aucune modification du texte, mais sous un autre titre La Rivolta dei santi maledetti). La version de 1923 représente un second stade du texte. Le lexique trop marxisant est effacé, et une longue préface non dénuée d'intérêt (elle rappelle le contexte autobiographique de l'écriture du livre) mais écrite dans un style inutilement verbeux et ampoulé à l'image du titre (Ritratto delle cose d'Italia, degli eroi, del popolo, degli avvenimenti, delle esperienze e inquietudini della nostra generazione) tente de faire de la "révolution caporettiste » un élément précurseur et moteur de la révolution fasciste. Cette version est certes un indispensable document historique, mais elle n'est pas le texte. Bref, pour comprendre Viva Caporetto!, il faut d'abord établir une distinction nette entre la version originale de 1921 et la version de 1923, adaptée par son auteur au moment où il venait de faire le choix du fascisme en septembre 1922. Leur contexte de publication est tout aussi déroutant : le livre est publié en 1921 aux éditions « Oceanica » du nom du mouvement et de la revue que Malaparte vient de créer en s'inspirant des groupes "Clarté » de Barbusse et la version "fascisée » de 1923 à nouveau éditée par les éditions de Guglielmo Lucidi qui continue de publier toute la production pacifiste d'après-guerre. Ces complexités éditoriales et idéologiques sont encore accrues par la légende d'un auteur caméléon, qui naît avec ce premier livre et qui ne cessera en retour d'augmenter la méfiance vis-à-vis d'un texte à qui l'on prête la même versatilité qu'à son auteur. Car face à un objet aussi insaisissable, aussi irréductible à toute tentative d'identification, on a tôt fait d'en maîtriser la complexité en lui collant les étiquettes habituelles, «malapartiste », " provocateur », ou encore la séduisante - mais bien malheureuse formule de "subversivité réversible ", forgée par Mario Isnenghi, faussant ainsi la portée d'un témoignage de guerre authentique et sincère et risquant d'étouffer le souffle d'un premier livre qui est le cœur vivant de toute l'œuvre malapartienne ${ }^{3}$.

3 Car le malentendu Malaparte commence avec ce livre, dès ses premiers mots. On a voulu y voir le sceau d'une provocation juvénile, plutôt que la lucidité d'un jeune écrivain prématurément mûri par la tranchée, par l'expérience de cinq années de guerre, creuset de l'homme et de l'œuvre. Or, Malaparte ${ }^{4}$ est déjà tout entier dans cette première page : l'isolement de la voix, l'écriture comme un chant qui libère l'amour et la haine intimement mêlés, une religion de vie bâtie sur l'expérience de la guerre, expérience physique et mystique de communion de l'homme avec la terre, d'une " primitivité » retrouvée ${ }^{5}$.

Ce malentendu tient moins au contenu du livre qu'à son irrésistible force de résistance et d'opposition, car en vérité le propos de Viva Caporetto! est, aux deux stades du texte, simple et univoque : il s'agit de réhabiliter le courage et le sacrifice des soldats contre le mépris des autorités militaires. Le fait que Malaparte ait "utilisé » son livre, à trois reprises, pour entrer sur la scène professionnelle et culturelle italienne n'infirme en rien l'authenticité du propos. Viva Caporetto! est le livre d'un soldat avant d'être le livre d'un intellectuel. Il est tout d'abord une réponse directe et explicite au Bulletin militaire rédigé par le général en chef des armées Luigi Cadorna, quelques heures après la déroute de Caporetto (bulletin aussitôt corrigé par le gouvernement, alors que la première version avait déjà été diffusée en Italie et à l'étranger), qui rend les soldats des régiments la $\mathrm{II}^{\mathrm{e}}$ Armée responsables de la percée autrichienne et les accuse publiquement de « lâcheté » :

La mancata resistenza di riparti della $\mathrm{II}^{\mathrm{a}}$ Armata vilmente ritiratisi senza combattere, o ignominiosamente arresisi al nemico, ha permesso alle forze austro germaniche di rompere la nostra ala sinistra sulla fronte Giulia. Gli sforzi valorosi 
delle altre truppe non sono riusciti ad impedire all'avversario di penetrare nel sacro

suolo della Patria. (Bulletin, 28 octobre 1917)

Lorsque Malaparte écrit Viva Caporetto!, au cours de l'hiver 1919, dans un village des Flandres où sa compagnie est en attente de démobilisation, la commission d'enquête parlementaire n'a pas rendu ses conclusions sur la responsabilité avérée des autorités militaires italiennes, ni les historiens leurs analyses des conséquences paradoxales de cette déroute qui permit, grâce au remplacement des chefs et à un meilleur traitement des troupes, de mettre fin à des choix de stratégie absurdes et anachroniques et qui porta donc à la victoire finale. Mais Malaparte a suffisamment d'expérience du terrain et des commandements militaires pour avoir compris les causes militaires, l'ingéniosité du plan ennemi, le contexte météorologique et géographique défavorable, et les erreurs humaines qui ont conduit à Caporetto. Et il a été le témoin direct de l'amélioration du traitement des soldats aussi bien que des répercussions sur leur psychologie et leur volonté - à Bligny par exemple - de laver l'honneur floué. Ce n'est donc pas l'événement militaire en soi, ni ses conséquences qui intéressent Malaparte mais sa portée historique et symbolique. Le " retournement » qu'il tente d'opérer, n'est en rien la pirouette d'un jeune auteur provocateur mais une entreprise de réhabilitation, comprise comme le prolongement de sa mission de soldat, d'officier, et de rapporteur militaire - ne vient-il pas au moment d'écrire Viva Caporetto! de rédiger un rapport sur les actions de sa compagnie à Bligny pour le général Albricci ${ }^{6} ?$ - comme un devoir visà-vis des soldats qu'il a connus et aimés, qu'il a commandés et vu mourir. C'est à Caporetto que les autorités militaires ont sali l'honneur des combattants et bafoué la mémoire des morts, c'est à Caporetto que doit être reconstruite cette mémoire et restauré cet honneur. Malaparte est très conscient de la difficulté de ce qu'il entreprend. Il n'est pas un ingénu, il a combattu à Bligny en avril 1918 avec des troupes italiennes qui ont tout fait pour repousser l'avancée allemande, il y a vu mourir 5000 soldats d'une armée prête à tout pour prouver le courage que ses propres chefs lui avaient nié, il fait partie des jeunes soldats affectés à l'organisation des conférences pour la paix à Versailles, il sait fort bien la puissance dévastatrice et contre-productive du simple nom de Caporetto : ce nom résonne comme un scandale dans un pays meurtri par la guerre. L'Italie tente alors de se reconstruire dans un difficile contexte de pourparlers entre pays victorieux et ces derniers ne font pas la part belle à un allié capricieux, qui s'est donné le temps de choisir son camp et qui a le toupet de demander le respect scrupuleux des clauses du traité de Londres. Malaparte sait que le gouvernement italien tente à tout prix d'évacuer la mémoire honteuse de la gigantesque déroute, en érigeant des monuments aux morts pour la Patrie, en désignant un Soldat inconnu et en reconstruisant une cartographie glorieuse des grandes batailles, avec pour point d'orgue la facile victoire de Vittorio Veneto face à l'armée d'un Empire austro-hongrois en train de se défaire.

6 Mais le jeune soldat-écrivain est convaincu de devoir dénoncer un scandale bien plus grand: le déshonneur jeté sur les soldats de la $\mathrm{II}^{\mathrm{e}}$ Armée et au-delà, la mort sans sépulture ni reconnaissance de centaines de milliers de soldats. C'est une mission qu'il prend en charge au nom de tous ses compagnons morts, au nom aussi de ceux qui ne peuvent ni ne veulent parler de la guerre, de leur guerre: «I fanti, in gran parte analfabeti, non scrivevano mai: oppure, se dettavano al compagno, più istruito, una cartolina in franchigia, non parlavano mai di guerra ${ }^{7}$. » Le soldat Suckert est celui qui écrit, «l'uomo che scrive ${ }^{8} »$. 
7 Pour témoigner, parler à la place des simples soldats, Malaparte se débarrasse de toutes ses médailles, dès les premières pages de Viva Caporetto! Il est semblable aux hommes « humbles et primitifs » dont il partagea le pain et la paille, un « uomo qualunque », un « uomo normale », un uomo in carne ed ossa ${ }^{9}$ ».

8 Le parcours de Kurt Erich Suckert ne ressemble pourtant en rien à celui d'un soldat ordinaire, et pas seulement parce qu'il fut, tout jeune élève du lycée Cicognini de Prato, de nationalité allemande par son père, secrétaire de la section jeunesse du parti républicain de sa ville, interventionniste de la première heure aux côtés des Français, parti dès l'automne 1914 avec la Légion garibaldienne qui combat en Argonne. Démobilisé en mars 1915, il est volontaire deux mois plus tard, soldat de $2^{\mathrm{e}}$ classe de la Brigade des Alpes. En juillet, il est lanceur de grenades en première ligne, en octobre 1915 participe aux assauts du Col di Lana. Au cours de l'été 1917, il est nommé sous-lieutenant, en octobre de la même année, au moment de Caporetto, sa brigade est appelée pour contenir la brèche ouverte par les troupes autrichiennes et la débâcle des troupes italiennes. Puis il retourne combattre en première ligne sur le Grappa puis sur le Tomba. En juin 1918, après deux mois d'instruction à Saint-Ouen, il est envoyé au sud-ouest de Reims, entre le Bois des Éclisses et la "montagne " de Bligny, au commandement de la $94^{\mathrm{e}}$ section d'assaut lance-flammes de la Brigade des Alpes, avec le général Albricci. Français et Italiens repoussent les Allemands qui menaçaient Paris. Pris dans une attaque au gaz, il est blessé aux poumons. Il obtient la croix de guerre française avec palmes (juillet 1918) pour avoir conduit en tant que « commandant de la section lance-flammes, sous un feu nourri de mitrailleuse, sa propre troupe à l'assaut de fortes positions ennemies, faisant preuve d'un calme et d'un courage hors du commun ». Côté italien, lui seront encore décernées, le 16 août 1918, la "médaille d'argent à la valeur militaire ", le 27 août 1918, la croix de guerre, tandis que le $52^{\text {e }}$ de la Brigade des Alpes reçoit la médaille d'or. Côté français, le 11 janvier 1919, il reçoit aussi une citation à l'ordre du régiment par le général Guillaumat ( $5^{\mathrm{e}}$ armée) en tant qu'« officier de grande valeur ${ }^{10}$ ».

9 Malaparte, dans ses écrits, ne fait jamais mention de ses médailles. Seule la correspondance contient quelques rapides allusions : « Ho delle medaglie, ho una ferita, ho quattro anni di vera guerra, ho la coscienza a posto e fo quel che mi pare. Mi meraviglia che Prezzolini abbia scambiato questa mia libertà orgogliosa per il solito disfattismo di molti proletari e di molti borghesi ${ }^{11}$." Comment comprendre qu'un soldat de son cran se revendique caporettista et jette ses médailles aux va-nu-pieds de Caporetto ? Comment comprendre qu'un jeune officier, héros de guerre, gazé, plurimédaillé sur les deux fronts ne s'intéresse qu'aux "forçats de la guerre ", aux "galériens des tranchées », aux " condamnés », aux " parias », à la " piétaille ", aux " galeux ", aux " pouilleux », à la « chair à canon des onze batailles », aux « va-nu-pieds du Karst», au "peuple des tranchées »? À un moment où commence déjà de se construire le mythe du vittoriovenetismo qui permettra au fascisme de nourrir sa rhétorique de la Grande Guerre en effaçant (pour plusieurs décennies) la mémoire historique de celle-ci, ces histoires qui sentent la boue et le sang, risquent de raviver la douleur d'un pays en deuil, et d'attiser la question tabou de la justification des massacres. Et surtout, le mépris et l'ignorance de l'arrière, que Viva Caporetto! dénonce, est loin d'avoir pris fin avec la guerre. Peut-on parler de la « haine de la Nation » à cette même Nation qui a voulu la guerre et entretenu un discours interventionniste et patriotique sans la faire elle-même. Viva Caporetto! s'adresse à ceux qu'il accuse, aux 
"bourgeois », aux " embusqués », aux " décorés de la zone tempérée », aux "patriotes des angles morts et de l'arrière ». Malaparte avait-il vraiment l'intention de se faire comprendre en lançant une telle grenade? Une seule chose importait : témoigner et dire la vérité.

La vérité de la guerre que raconte Malaparte, le choc des tranchées, la folie des soldats, le mépris des supérieurs pour les vies humaines, l'absurdité des choix stratégiques, les justifications rhétoriques de massacres inutiles, l'indifférence assassine de l'arrière, nous est connue aujourd'hui. Qui, hormis les fantassins, acceptait alors de voir cette sale guerre en face? Il y eut, bien sûr, les médecins et psychiatres, qui durent soigner les symptômes liés à la névrose de guerre (ce qu'on appelle aujourd'hui syndrome de stress post-traumatique ${ }^{12}$ ). Du côté des écrivains, on pense immédiatement à Céline, mais le nihilisme de Bardamu est tout à fait étranger à l'hommage vibrant que le soldat Suckert rend à ses compagnons, bien plus proche en cela, du Jünger d'Orages d'acier. En Italie, les témoignages des écrivains et poètes, tels que Giovanni Boine et Clemente Rebora, que la guerre avait rendus fous ou malades, sont longtemps restés inédits ${ }^{13}$. Quant aux indispensables récits de guerre de Carlo Salsa ou Emilio Lussu (Trincee. Confidenze di un fante, 1924 et Un anno sull'altipiano, 1945), ils n'eurent au moment de leur parution que peu de lecteurs. Comme c'est le cas pour Viva Caporetto! rares étaient ceux qui pouvaient lire ces livres. Il aurait fallu pour cela s'émanciper d'une histoire de la Grande Guerre, encore empreinte de rhétorique (interventionniste, fasciste, nationaliste, ou simplement patriotique, c'est-à-dire grevée par le complexe de la Nation victorieuse et «mutilée»). Lorsque le premier biographe de Malaparte écrit, en 1980 : «E c'è da credere che un libro simile avrebbe pessima accoglienza anche oggi, visto che la retorica della prima guerra mondiale è ancora molto viva ${ }^{14} "$ " c'est on ne peut plus vrai. Rappelons d'ailleurs qu'à cette même époque les contemporains de Guerri connaissent mieux Caporetto par Il giornale di guerra e di prigionia de Carlo Emilio Gadda, dont le style l'emporte en mépris sur la langue des communiqués de Cadorna ${ }^{15}$. Il faut attendre les années 1970, la guerre du Vietnam, une opinion publique sensibilisée aux questions pacifistes et antimilitaristes, et surtout la fin du siècle, quand l'onde de choc des horreurs de la Seconde Guerre mondiale se sera estompée et qu'une génération d'historiens britanniques, américains, français, italiens marqueront la continuité $\mathrm{du} \mathrm{xx}^{\mathrm{e}}$ siècle européen, pour que ces rares et incontournables récits de guerre soient diffusés et connus... au prix parfois, d'une interprétation anachronique et d'un malentendu qui n'est pas levé aujourd'hui! Le livre de guerre de Lussu (contrairement à l'adaptation cinématographique antimilitariste qu'en donne Francesco Rosi en 1970) condamne la façon dont elle a été menée, la façon dont ont été traités les soldats et, par conséquent, interroge également les raisons qui ont conduit à la guerre, mais ne condamne jamais explicitement les raisons de l'intervention : Lussu laissera en suspens la question de son propre interventionnisme. Il existe aujourd'hui un quiproquo de même nature avec Viva Caporetto! qui est un ouvrage contre les abus de l'autorité des commandements en guerre, contre la rhétorique de guerre, contre les décisions d'un gouvernement en guerre, contre le mépris de l'arrière, mais ce n'est pas un livre contre la guerre, même si la réflexion sur l'absurdité de la guerre de tranchées, les inutiles assauts contre barbelés et obus mènent bien des fois Malaparte à interroger les raisons de la guerre.

11 Malaparte alors âgé de vingt-trois ans s'attaque, en même temps qu'aux autorités militaire et politique, à une société tout entière. Pour prendre la mesure de ce contre quoi se bat le jeune Malaparte en tentant de réhabiliter la gloire du fante, il faut se 
souvenir de l'événement le plus emblématique et paroxystique du mépris où a été tenu le soldat italien - en qui les commandements n'ont toujours vu qu'un déserteur potentiel : la mort de cent mille soldats prisonniers, laissés mourir de faim et de froid par le gouvernement italien dans les camps autrichiens et allemands. Ces faits n'ont été exhumés qu'à la toute fin $\mathrm{du} \mathrm{xx}^{\mathrm{e}}$ siècle avec les travaux de l'historienne Giovanna Procacci ${ }^{16}$. Le fait qu'un tué sur sept de la guerre de 1915-1918 soit mort de faim en prison (une proportion jamais atteinte dans les autres armées alliées, qui ont respecté le protocole de traitement des prisonniers, avec autorisation de circulation des trains de la Croix-Rouge, par exemple) est un fait encore aujourd'hui presque absent de la mémoire nationale. On comprend à quel point Malaparte est isolé : ce ne sont pas seulement les ministres de l'Intérieur de trois gouvernements successifs qui ont censuré son livre entre 1921 et 1923 , c'est une chape de silence qui dura près de soixante ans (la première réédition, celle de Falqui en 1961, présenta la version de 1923 parmi d'autres écrits de la période fasciste ; il faut attendre l'édition de Mario Isnenghi en 1980 pour que la parole de Viva Caporetto! soit entendue).

Rappelons enfin que la réhabilitation des fusillés pour l'exemple, symboles et martyrs de l'arbitraire des lois martiales, est aujourd'hui encore objet de débat, en France comme en Italie. On se souvient qu'il a fallu attendre 1998 en France pour que soit seulement évoquée la possible réhabilitation des mutins de 1917 par Lionel Jospin alors Premier ministre, qui souhaita dans son discours de Craonne, que les fusillés pour l'exemple fussent « réintégrés dans la mémoire collective nationale ». Mais aujourd'hui encore, 18 ans après le discours de Craonne, le projet de loi sur la réhabilitation collective des fusillés pour l'exemple (et inscription de leurs noms sur les monuments aux morts) fait toujours débat et n'a toujours pas été voté. En Italie le député du Parti démocratique Gian Paolo Scanu a fait approuver le 21 mai 2015 (à 100 ans presque jour pour jour de l'entrée en guerre de l'Italie) par le parlement, une loi en faveur de la réhabilitation des "tués oubliés" (caduti dimenticati), avec projet de scellement d'une plaque à la mémoire des fusillés sur les murs du Vittoriano à Rome (où se trouve le tombeau du Soldat inconnu italien), révision des procès de justice sommaire et récupération des grades. La loi est actuellement soumise au Sénat.

\section{Le tourment d'une génération}

13 La guerre fut pour Malaparte une "expérience de mutation spirituelle profonde ${ }^{17}$ ", une "transformation intime ${ }^{18}$ ", tout à la fois seconde naissance, éveil politique, traumatisme et maturation psychiques, initiation et révélation mystique. Ce n'est qu'avec son dernier roman écrit entre 1951 et 1952, Mamma marcia, fragmentaire et inachevé, qu'il réussira à dire simplement, sans le truchement de la parole politique, en laissant de côté sa mission de porte-parole des fantassins, ce qu'a été la guerre pour lui : un voyage de son vivant dans le royaume des morts, et « l'obscur, profond, inconscient souvenir de la guerre ", de ses horreurs et de ses heures merveilleuses, qui ne le quitte plus $^{19}$. Toute l'œuvre de Malaparte est marquée par ce souvenir, comme si l'écriture n'avait cessé de traquer cette expérience humaine indicible, une expérience de solitude intense, aux limites de la folie.

En 1921, le premier terme qui définit cette expérience est « désespoir ». La nature de ce désespoir est physique autant que psychique. Celui qui survit aux obus, aux balles des 
mitrailleuses et à l'effondrement de tous ses repères et certitudes, doit faire face à une soudaine et insoutenable lucidité.

L'uomo che per la prima volta entra nel cerchio della guerra, deve ricominciare, da capo, a imparare il mondo: egli scopre, a un tratto, da un giorno all'altro, che le parole non si appropriano alle cose e che la vita della natura è diversa da quella che i vocaboli indicano e che la consuetudine giornaliera gli ha dato a credere. Egli comincia a pensare, per la prima volta ${ }^{20}$.

La perte des repères et du langage n'est que le premier choc. L'indifférence des chefs et de l'arrière en constituent un deuxième, non moindre :

Tutto ciò che significava eccitamento alla guerra senza scapito personale, apparve agli occhi del fante come una forma di sfruttamento della sua miseria eroica, come un comodo sistema di patriottismo attivo, come un insulto fatto ai morti e ai condannati a morte.

Comitati di soccorso, Case del soldato, Associazioni patriottiche, Comitati di beneficenza, tutto ciò gli aveva l'aria di una presa in giro, di un «bosco elegante e umanitario", di una enorme associazione dalle innumerevoli filiali intesa a gabbarlo, a fargli digerire l'amara pillola della guerra con l'aiuto di uno zuccherino tricolore, a fargli dimenticare gli orrori della trincea con l'esibizione di signorine in soggòlo, di bandierine interalleate e di cartoline illustrate ${ }^{21}$.

Dans la première version écrite à chaud entre 1919 et 1920, Malaparte ne nomme jamais directement ce "désespoir», (parfois "presa di coscienza», "presa in giro " ou "beffa ») mais projette immédiatement celui-ci dans la haine, le sentiment d'injustice qui en découle. C'est dans la version de 1923, dans le long Ritratto fascisant, puis dans L'Europa vivente, où est théorisé la même année le premier fascisme comme "syndicalisme national», que Malaparte fait allusion à un autre sentiment qui alimente ce "désespoir ", à savoir l'état de stupeur et de mélancolie douloureuse qui a suivi les années de guerre: le «tourment» de la génération d'après-guerre, "l'inquiétude subtile», dont souffrent les survivants, anciens combattants des tranchées et plus largement tous les «survivants» du cataclysme, rongés, consciemment ou inconsciemment, par la mémoire des horreurs subies et infligées, et par le doute que cela n'ait servi à rien.

Ma tutto, allora, aveva una ragione profonda in noi stessi, una ragione di rapporto con i fatti e con lo spirito di allora, una giustificazione in noi e fuori di noi. Visti ora e di qui, da questo tempo e da questo luogo, da queste circostanze e da queste necessità, le cose di quella viva e pur ordinaria stagione ci sembrano essere state impossibili e arbitrarie anche allora, come se più non le intendiamo.

[...] Il sospetto che gli anni trascorsi (liberamente per me, ch'ero volontario di guerra) nell'infernale e gloriosissimo fango delle fosse carsiche, avessero qualcosa in loro d'arbitrario, in rapporto al nuovo spinto eroico già nascente dal pacificarsi del tumulto guerresco, mi dava spesso un'inquietudine sottile, che voltava talvolta, all'improvviso, in ira. Ma quel che mi dominava in quella stagione era la fiducia che avevo in un propizio svolgimento delle cose in Italia. Una lieta sicurezza di quel che i prossimi avvenimenti avrebbero portato in aiuto dello spirito rivoluzionario ed eroico della nostra generazione ${ }^{22}$.

17 Comme beaucoup de sa génération, comme de très nombreux autres reduci, terme que le français ancien combattant rend assez mal, et que rend très bien en revanche celui de survivant, tel que le mot sera employé après l'autre guerre, par Primo Levi par exemple, survivant de l'horreur des camps, et de la terreur de ne pouvoir en rapporter le témoignage. C'est par ce biais précisément que Viva Caporetto! s'inscrit dans le contexte du fascisme naissant de 1923 ; sa lecture de la guerre est certes éloignée du mythe de la Grande Guerre que construira le fascisme, mais elle n'est pas éloignée de l'état d'esprit 
qui anime les premiers squadristes en quête d'une révolution; il est le livre d'une génération de survivants, qui doit survivre coûte que coûte, à l'horreur et au doute. Même si les épanchements sont retenus dans cette version déjà imprégnée de lexique fascisant (la boue est "très glorieuse", les tranchées sont anoblies en "fossés du Karst »), l'impulsion à la rébellion est réelle, nourrie par la foi du survivant, dans la continuité immédiate du combat, par la conviction intime (chez qui a survécu à la mort mais aussi à la perte des repères, à la conscience de soi comme assassin) que rien ne pourra plus être comme avant. En 1923, Viva Caporetto! est avant tout un moyen de poursuivre la guerre pour un soldat démobilisé qui n'a plus de repères dans un monde sans guerre.

En 1946, la "maladie de la guerre », en réalité mal de l'après-guerre, «tourment » et « inquiétude » d'une génération, est froidement appelée le «fatto guerra ». Dans le Memoriale écrit en 1946 pour sa défense devant les tribunaux de l'épuration, Malaparte donne une analyse lucide de ces années-là et invoque la guerre comme ligne de partage entre sa génération et celle, plus jeune de trois années et qui n'a pas connu directement la tranchée, de Piero Gobetti :

Questi attacchi da parte dei giornali fascisti m'irritarono e accentuarono la mia tendenza a orientarmi verso gli elementi intellettuali dell'antifascismo, dai quali tuttavia mi divideva, in molti casi, una diversa valutazione della guerra, delle sue premesse e delle sue conseguenze. Io rimanevo legato al fatto "guerra», che era stata la più valida, rigorosa e impegnativa esperienza della mia gioventù e la più sinceramente sofferta. V'accenno ora, di sfuggita, perché è tale la mia fedeltà al fatto guerra, che mi ha spinto, nel Settembre 1922, dopo la crisi sociale e politica italiana dell'Agosto 1922 (sciopero legalitario), a iscrivermi al Partito fascista.

[...] Passavamo lunghe ore insieme a discutere ogni sorta di problemi sociali, letterarii, politici, religiosi. Su un punto solo non eravamo d'accordo: sulla guerra, egli [Piero Gobetti] svalutava l'importanza morale della guerra per le giovani generazioni che vi avevano preso parte, io, forse, la sopravalutavo. Egli era più giovane di me, non aveva partecipato alla guerra, e perciò era molto più freddo, più sereno, più obbiettivo di fronte al dramma della guerra. Era anche molto più libero nei suoi giudizi, poiché non era impacciato e appesantito dalla retorica patriottica di noi reduci. La guerra per me era una mia tradizione personale, la mia prima fondamentale esperienza di vita. Non potevo, perciò, essere obbiettivo, né libero di fronte alla guerra. Ed è appunto il fatto "guerra» che mi ha impedito di essere un antifascista, allora ${ }^{23}$.

Ce n'est pas parce que Malaparte ne dit pas toute la vérité (il ne s'est pas inscrit au Faisceau de Florence en réaction immédiate aux grèves du mois d'août, ni pour travailler au sein des organisations syndicales fascistes, mais son travail auprès des ouvriers a fait suite à son adhésion et a certes satisfait son désir d'occuper une fonction ${ }^{24}$, d'avoir un titre, comme il avait eu plusieurs fonctions et grades successifs dans l'armée), ni parce qu'il use sans vergogne de litotes qui font sourire, que ses propos perdent toute authenticité. Ces pages, bien au contraire, disent au plus juste le pourquoi d'une adhésion au fascisme dont la clé est à chercher dans l'expérience de la guerre et nous questionnent à nouveau sur le risque d'une lecture erronée de Viva Caporetto!, critique de la guerre depuis l'intérieur de la guerre, avec le plus grand respect pour les troupes, un goût du combat, une foi en la mystique de la guerre comme possibilité d'accès à un infini surhumain et une haine féroce pour ceux qui ont bafoué cette " religion ». Ce n'est pas un livre antimilitariste. Malgré un langage emprunté à la contemporaine révolution bolchevique (" prolétaires ", " grève militaire »), ce n'est pas non plus un ouvrage pacifiste. On peut comprendre alors que Malaparte ait cru possible 
de détourner l'attention des étudiants squadristes qui détruisaient à Rome les vitrines des libraires et de lever le malentendu en "fascisant » le titre de son opuscule dès mars-avril 1921. Ces quelques mots à Bino Binazzi sont éclairants sur la place négligeable qu'accorde alors Malaparte au mouvement fasciste sur l'échelle de ses propres valeurs et de sa propre expérience : "Così nella recensione che ella mi farà, parli del libro come se il titolo fosse il nuovo, e accenni al fatto fascista. Inteso? $\mathrm{E} \mathrm{mi}$ risponda subito per dirmi il suo parere in merito al nuovo titolo ${ }^{25}$.» En 1921 déjà, Malaparte est prêt à utiliser le « fait fasciste » comme un laisser-passer pour son livre ; deux ans plus tard, tandis que l'engagement politique de l'auteur est conscient et assumé, de nombreuses corrections du texte résulteront d'un semblable processus de maquillage.

Avant de juger que Malaparte manipule l'histoire à son gré comme l'ont dit et répété biographes et commentateurs, il est par conséquent nécessaire de considérer le contexte de falsification rhétorique et de manipulation historique contre lequel Malaparte se bat et sur lequel le jeune soldat-écrivain fonde sa mission: dire la vérité de la guerre contre l'histoire officielle, parler pour ceux qui ne le peuvent pas, parler contre les autorités, ouvrir la voie à des énergies nouvelles créées par la guerre et leur trouver un nom. Les écrits de ces années d'après-guerre sont très loin d'être aussi réussis que Viva Caporetto!, mais ils témoignent du combat intellectuel du jeune Malaparte, de l'énergie qu'il déploie, de la foi qui l'anime et de la difficulté de se faire une place au sein d'un espace idéologique européen trop étroit, déjà miné par sa bipolarité, et qui «sous-évalue» le "fait guerre». Il invente donc tour à tour le mouvement océanique avec la revue Oceanica (janvier-mars 1921) et une théorie du fascisme comme réinterprétation du syndicalisme révolutionnaire de Sorel avec L'Europa vivente (1923), non sans avoir appelé dans Le nozze degli eunuchi (1922) à la création d'un nouvel "art latin » et jugé les écrivains les plus en vue de son époque incapables de le représenter. Malaparte est isolé, il en est conscient et croit possible - l'illusion durera quelques années - d'absorber cette solitude dans l'adhésion au fascisme.

Noi eravamo tra quei fanti, primi fra i primi, sempre; soldati della rivoluzione. Abbiamo sofferto e insegnato come si soffre. Più tardi, dopo il ritorno, sicuri e superbi del nostro onore di fanti e delle nostre ferite, siamo stati soli a difendere l'onore e la dignità del popolo innocente e implacabile che aveva vinto cento battaglie, e s'era rivoltato a Caporetto non già contro l'Italia, contro il vivente spirito della Patria, ma contro i bestemmiatori dei morti e gli schernitori del sacrificio dei vivi. Siamo stati soli, quando tutti modulavan sui flauti magici e sugli organetti di Barberia la terribile imprecazione di Cadorna, siamo stati soli a mostrare che la rivolta di Caporetto non era stata né un tradimento né una fuga, ma una selvaggia e orrenda sollevazione dei fanti, del popolo delle trincee, dei soldati della rivoluzione nazionale, contro chi s'era tenuto lontano dalla guerra bestemmiando il sangue versato, i sacrifici sofferti, la gloria e l'orgoglio della nostra passione ${ }^{26}$.

21 Viva Caporetto! n'est pas un ouvrage historique, et c'est pourquoi il est le plus réussi des écrits de ces années. Dans une période où il est devenu impossible d'écrire avec les mots et la syntaxe disponibles, Malaparte écrit une sorte de fable, une parabole politique et sociale au plus près de la véracité des faits historiques, mais inscrits dans une narration fabuleuse et mythique, à son tour témoin de la dimension transcendante et symbolique des événements vécus. Le récit de guerre est parcouru d'expressions qui opposent à la folie des tranchées une dimension humaine possible: «l'inexplicable 
nécessité de la souffrance ", l'« infini inhumain », l'« infini humain », l'« insondable mystère du sacrifice ", le " frisson » face à la « fatalité » et un espace, où chaque homme est soumis à l'étau du « cercle de la guerre », où vie et mort s'équivalent :

Tra uomo e uomo, v'era lo spazio lasciato da un morto. Altri avrebbero lasciati altri spazi.

[...] Poi di nuovo, l'ombra della notte immutabile e dovunque presente riempiva gli spazi e intervalli. Tra uomo ed uomo qualcuno si sedeva. Il solito brivido - il brivido dell'infinito - passava da cuore a cuore e chiudeva il cerchio ${ }^{27}$. D'Annunzio, paru la même année que le livre de Malaparte ${ }^{28}$. Notturno fut rédigé cinq ans plus tôt, dès l'année 1916, par le poète qui vient de perdre un œil dans un accident et qui guide sa plume sur des bandelettes de papier. Entre la date de rédaction et la date de parution de Notturno, le poète occupe la ville de Fiume, en protestation contre la «victoire mutilée » du traité de Versailles (il forge lui-même les termes de cette métaphore puissante sur la première page du Corriere d'Italia du 24 octobre 1918). À ce moment, D'Annunzio vient d'achever, par le travail d'écriture, le deuil de son œil droit, et interprète sa propre mutilation comme un don à la Patrie, supérieur au don de la vie :

L'ispirazione dell'eroismo soffia anche su gli invalidi, soffia anche su i tronconi umani, soffia anche su le carcasse impotenti.

Ti racconto una cosa grande, a te che dovevi ricondurre in patria il mio corpo sacrificato.

Siamo senz'ali. C'è una gloria dell'alto e c'è una gloria del profondo. C'è una morte bella e c’è una morte ancor più bella ${ }^{29}$.

La mutilation est ici une forme de sacrifice symbolique qui contribue à l'intégration de l'individu à l'histoire, et de l'histoire au mythe. Un an avant l'avènement du fascisme, Notturno redonnait à une génération coupée - comme une branche est coupée - le lien à son passé, en faisant des morts de la guerre la grandeur de celle-ci, et le lien à son futur, en faisant du territoire national un corps à préserver dans son intégrité, fondant ainsi, dans le prolongement métaphorique de la guerre, l'intégrité de la Nation sur la mutilation des individus.

Malaparte fait exactement la même chose: Caporetto la gigantesque déroute, l'effondrement du front oriental devient la glorieuse mémoire des soldats, des vivants comme des morts. Caporetto la brêche, la partie perdue de territoire devient la médaille à épingler sur les haillons des soldats du Karst.

\section{Fantassins, vagabonds et chemises noires}

Le fantassin couvert de loques est le protagoniste du récit que bâtit Malaparte. Au centre de la parabole se tient le gueux, le va-nu-pieds, le vagabond. Presque à chaque page, le fantassin, le pouilleux de Caporetto, est érigé en figure allégorique assez puissante pour défaire la Victoire des cartes postales illustrées. Dans le prolongement 
immédiat du livre de guerre, cette figure renaît, en janvier 1921, sous les traits de l'« homme océanique ».

Il est donc important dans le contexte mouvant de ces années, et dans l'itinéraire non moins fuyant de Malaparte, au moment de l'après-guerre, entre l'année 1919 où il commence à rédiger Viva Caporetto! et l'année 1923 où il collabore assidument à La Conquista dello Stato et écrit L'Europa vivente, de rappeler l'importance du bref et intense moment océanique, c'est-à-dire les trois mois où le jeune officier tout juste démobilisé et libéré de sa mission diplomatique à Varsovie en décembre 1920, choisit de lancer sa carrière intellectuelle à Rome avec pour viatique, en même temps que la publication de Viva Caporetto!, le lancement d'un mouvement spirituel international dont il est l'unique adepte : l'océanisme. La première version de 1921 est intimement liée, dans son style et sa langue "océaniques", et dans sa forme éditoriale (le frontispice porte mention des "Éditions de l'Oceanica») à la création de la revue Oceanica, première expérience, certes éphémère et maladroite, de journalisme culturel de Malaparte ${ }^{30}$. Quant aux deux versions suivantes de Viva caporetto! (avril 1921 et 1923), non seulement le style des premier et dernier chapitres est encore imprégné du souffle océanique, mais elles sont publiées par la Rassegna internazionale et estampillées en première de couverture du sigle U.D.C., du nom de l'association pacifiste et humanitaire britannique Union of Democratic Control, dont le conte Guglielmo Lucidi, directeur de la Rassegna, parrain de la jeune revue pacifiste Oceanica, est le représentant en Italie. Le pamphlet de l'inconnu Curt Erich Suckert se retrouve alors publié, en avril 1921, dans une collection qui diffuse au même moment les programmes de Romain Rolland pour une "Internationale de la pensée ", le projet pacifiste et panhumaniste de Barbusse promu dans Une lueur dans l'abîme. Ce que veut le groupe «Clarté » (1920).

Cette période océanique s'accompagne d'une iconographie évocatrice de l'univers personnel de Malaparte et place la figure du Gueux sur le devant de la scène: la reproduction d'une gravure de Rembrandt, évoquant un vagabond (en réalité portrait de la mère du peintre), figure en couverture du Viva Caporetto! de février 1921. Cette icône issue de la revue Oceanica et du mouvement qu'elle soutient, apparaitt inséparable du vagabond océanique emprunté à la série des Gueux de Callot, par l'intermédiaire d'une édition illustrée des poèmes de Aloysius Bertrand que possède Malaparte (Gaspard de la nuit, Paris, La Sirène, 1920). Comme le fante de Caporetto, le Gueux océanique porte le «mouvement qui répond le mieux à la soif d'espace et d'infini qui travaille cette humanité tourmentée de l'après-guerre ${ }^{31}$ " avec un projet de créer des groupes "océaniques» («commune artistique», "groupe d'hommes libres», adeptes d'une « religion de vie et de beauté ») qui prennent les groupes "Clarté » pour modèle. C'est celui-ci qui fait écrire au jeune Suckert, alors presque unique rédacteur de la revue, qu'il est le "Barbusse italien »; la formule est souvent reprise hors contexte, mais il s'agit bien sûr du Barbusse de Une lueur dans l'abîme dont Malaparte s'inspire, alors qu'il n'a jamais revendiqué une quelconque parenté avec l'auteur du Feu.

Le Gueux, vagabond ou va-nu-pieds, est une figure archétypale où s'incarne le moi de Malaparte, le moi nouveau, issu de la tranchée. Il est le Survivant, par excellence, celui qui veut, dans le dernier chapitre de Viva Caporetto!, auquel fait écho le manifeste de l'océanisme, «Vivre! Vivre! Vivre! ». Le Gueux est le fantassin dégagé de la gangue rhétorique accumulée par les journaux pour tenter pendant quatre ans de masquer la boucherie. Il est le soldat mort pour la Patrie, trahie par elle, qui se relève, la tête haute et l'honneur sauf, au sein du " peuple des tranchées ». Il est celui qui s'extrait, enfin, du 
« cercle » mortifère de la guerre (Viva Caporetto! est ponctué d'expressions qui évoquent l'enfermement physique et surtout mental du soldat : « cerchio della guerra », « cerchio di fuoco", " cerchio della potenza dittatoriale di Cadorna», "spezzare il cerchio troppo stretto »).

Il y a dans cette figure héritée de la tradition culturelle européenne, héritée du plus tourmenté romantisme de Bertrand, l'expression d'une virginité primitive, d'une nouvelle naissance et d'un nouveau départ. Mis à nu par l'expérience de la guerre, dépouillé de ses anciennes croyances héritées de son éducation familiale, scolaire et livresque, Malaparte en 1917 renaît au monde en haillons faits des mêmes chiffons qui habillent les fantassins usés par la tranchée ${ }^{32}$. Le Gueux incarne la «transformation intime » du jeune soldat interventionniste, du soldat lycéen qui quitte la tranchée pour revenir passer ses examens de fin d'année, du sous-lieutenant poète de la Brigade des Alpes, en soldat aguerri, vétéran de plusieurs fronts, qui a frôlé mille fois la mort, qui a donné la mort mille fois, qui a vécu au milieu des corps mourants de ses compagnons et de ses ennemis. Fort de tout ce qu'il a perdu au cours d'une expérience proche d'un voyage dans l'autre monde, le Gueux est cet homme revenu du pays des morts, survivant de la déflagration collective et intime, aussi nu et puissant que le premier homme à la conquête du monde.

Il est aussi celui qui par-dessus ses loques, en septembre 1922, revêt la Chemise noire. La figure du Gueux inscrit le passage du caporettisme au fascisme dans la continuité de l'expérience de la guerre, quand on voudrait, Gobetti le premier, y voir une conversion incompréhensible ["Questa parentesi fascista, così confusa che hanno potuto accogliere anche Lei (proprio non me lo aspettavo), che ne è l'antitesi $\left.{ }^{33} »\right]$. Il s'agit pourtant du même héros révolutionnaire, le christique fante de La Rivolta de 1923, le vagabond, le primitif, l'homme de la terre, qui réapparaît dans tous les écrits de l'époque. Dans L'Europa vivente, il est le "soldat de la révolution nationale », ou bien dans le Ritratto, le « faux Dimitri » que Malaparte appelle de ses vœux :

M'ero persuaso che il popolo dei fanti, ritornando alle case pacifiche dopo il dramma antico ed eroico della guerra, (o della terra?) avrebbe inevitabilmente compiuto la sua rivoluzione. [...] Ma sarebbe stato necessario qualcuno che guidasse il popolo dei fanti, un «falso Dimitri» che sommovesse le loro coscienze, pacificate dal glorioso sacrificio della guerra, con tutte le arti della retorica e della finzione; che andasse pellegrinando di paese in paese e di casa in casa a guarire i malati, a profetare, a compiere miracoli e ciarlatanerie d'ogni sorta. Sarebbe stato necessario una specie di santone, o di frataccio barbuto dagli stinchi nudi, una specie di eremita magro o di pastore lungo e patito, coperto di pelli di capra, con ciondoli medagliette buccie di serpi collane d'ossicini rosari di vetro stigmate cicatrici occhi luminosi e profondi, una specie di episcopo primitivo, di vescovo boschereccio che battezzasse i contadini in mezzo ai fiumi desse la benedizione ai campi dall'alto dei poggi con sue lunghe braccia lentissime, andasse predicando d'aia in aia la necessità di un ritorno a un cristianesimo naturale, terrestre, antimoderno, anticulturale, anticivile, sarebbe stato necessario, una specie di giovannibattista o di profeta che sollevasse il popolo dei fanti contadini, dei boscaioli, dei tanti pastori contro le città viziose, incancrenite d'oro, di carne e di lussuria, in nome di una giustizia agreste nemica del comune viver sociale, della scienza, della civiltà, della modernità; che guidasse l'esercito della santa vandea all'attacco delle città luminose in fondo all'orizzonte, nel nome di quel terribile «Cristo italiano» che da più di trecento anni la gente pacifica ha esiliato fra il popolo dei campi e dei poggi, come un nemico del beato vivere anticristiano e antieroico. Sarebbe stato necessario un uomo violento e fanatico, mistico e feroce, con un po' di sangue barbarico nelle vene tale da giustificar le necessarie imposture e gli estri matti, un «anabattista» della famiglia 
di Mûnzer, di Hubmeyer, di Hutter, di Giovanni Bécold detto Giovanni di Leyda, il sollevatore della plebe antipapale, l'antiluterano, il ribattezzatore, il banditore della nuova legge: «Qui non est natus ex aqua et spiritu sancto non potest intrare regnum coelorum». Un profeta o un ciarlatano, un cercatore di Dio, un rex plebis capace di camminare a piedi nudi innanzi alla folla, contro le città senza Dio, con la sacca da frate piena d'amuleti falsi e un crocefisso nelle mani ${ }^{34}$.

\section{L'expérience irréversible} sapposent de façon radicale que si on oublie la définition encore mal assuree, que Malaparte donne alors de ces termes. Il crée une foi et une idéologie personnelles avec la désinvolture et l'enthousiasme d'un tout jeune intellectuel en uniforme et il veut les partager, comme il a partagé le pain et la paille de ses soldats. La communion de la génération d'après-guerre par-delà les frontières et les idéologies ( « La vie est une seule et elle est internationale et la seule Internationale possible est vie », écrit-il dans le premier numéro d'Oceanica) ne se défait pas d'un enracinement national, ni d'une fierté italienne ${ }^{36}$. Une ambivalence qui agit de même dans ses propres convictions nationalistes et accompagne Malaparte au moment où s'élabore le choix de son adhésion au fascisme, puisqu'il propose à Gobetti, de fonder un syndicalisme international... nationaliste ${ }^{37}$ ! Même s'il s'agit d'une reconstruction a posteriori, formulée dans le souvenir d'un échange avec Gobetti pour le Memoriale de 1946, la sincérité et la conviction de Malaparte s'imposent par l'incongruité même de la proposition... et du destinataire. Ces utopies, encore vivantes et malléables, naissent chez Malaparte d'une volonté déjà enracinée de réformer la société décadente en profondeur et s'alimentent aux énergies vitales renées du sacrifice des fanti.

Malaparte tente de concilier et de contenir ces deux horizons dans l'espace d'élaboration que représentent les différentes versions de Viva Caporetto! et, contemporainement, de les mettre en acte par la création d'un mouvement spirituel (l'océanisme) et d'une doctrine idéologique (le Syndicalisme intégral). Sur le terrain politique, c'est un échec dont Malaparte tirera les fruits, peu à peu, au fil de ses expériences : dans son œuvre à venir, les questions politiques - avec un intérêt jamais démenti pour l'articulation entre national(isme) et international(isme) - seront prises en charge par l'écriture et évolueront dans un espace littéraire. Le politique s'accordera toujours plus intimement au poétique. Cet accord si particulier agit déjà dans Viva Caporetto! et La Rivolta dei santi maledetti, où le combattant et l'intellectuel s'affrontent, d'une version à l'autre et à l'intérieur de chacune, dans une bataille alors insoluble et 
qui ne trouvera d'issue que dans l'adhésion au fascisme avant d'évoluer sur des territoires politiques et littéraires toujours mieux assortis, et souvent tout aussi courageusement revendiqués.

Dans un passage autobiographique du Ritratto, Malaparte raconte comment il décide de quitter ses livres pour chasser avec les paysans de Saint-Hubert et les vieux soldats de sa compagnie. Il est conscient de la bataille que conduisent à l'intérieur de lui sa part cognitive et intellectuelle, celle du lettré, et sa part sensible, instinctive, " primitive ", celle du soldat et du révolté :

Quello che oggi, e non sempre, m'inquieta, allora mi spaventava. Quando mi accorsi che non potevo far nulla, né pensare, né ricordare, né credere, né sperare, senza una specie di fantasia ragionante, di cauta logica, di riguardoso abbandono, di sospetto vigilante e ossequiente; quando mi accorsi che l'umanista romantico aveva soffocato in me il fante, il cristianissimo fante lacero e spregiudicato, vivo e bestemmiatore, il fante che per quattro anni era vissuto nella mota, aveva pisciato nella stessa buca dove dormiva, s'era grattato la rogna, come un servo di Dio, in tutte le trincee delle Venezie, delle Argonne, di Reims, dello Chemin des Dames, s'era graffiato il viso e le mani a tutti i reticolati, aveva visto morire la gente intorno a lui, aveva urlato, pregato, sanguinato, aveva ucciso - anche ucciso; quando mi sentii mutare a poco a poco da quel che ero, tornar uomo, uomo sociale, uomo civile, uomo politico, uomo letterario, uomo moderno (ah! moderno), tornar falso, riguardoso, esperto, ipocrita, ragionante, da quell'uomo naturale, fisico, terrestre, semplice, primitivo, eroico e antico e umano che ero, (troppi aggettivi, troppi, o forse non bastano per rendere tutto il significato di fante); quando mi sentii avvilire, incattivire, immeschinire, mi ribellai, chiusi i libri, tornai ad imbrancarmi con i miei vecchi fanti contadini, boscaioli, cacciatori, pastori, via come prima per i boschi a inseguire cervi e cinghiali ${ }^{38}$.

L'adhésion au fascisme, qui fut suivi d'un intense travail de théorisation du «fascisme intégral " par l'écriture de plusieurs essais et une abondante collaboration à des journaux, fut sans doute perçue comme la solution pour faire vivre le fante. Mais mettre entre ses mains la plume du lettré a signifié alors une alliance forcée et monstrueuse : Malaparte étouffait son propre souffle.

Malaparte est né de la guerre "comme d'une seconde mère ${ }^{39}$ ", cette expérience l'a modelé corps et âme, et sa mémoire, tel un irréductible noyau, imprègne et inspire ses choix d'homme et d'écrivain d'un bout à l'autre de sa vie et de son œuvre, de Viva Caporetto! à Mamma marcia. Malaparte a tiré de son expérience un livre, qui n'est pas le récit de la guerre qu'il a vécu ${ }^{40}$, mais le récit d'une prise de conscience, d'une transformation psychique, psychologique et intellectuelle profonde, qui est en même temps l'arme avec laquelle il est déterminé à survivre au traumatisme, à prolonger le choc initiatique et à poursuivre le combat. Malaparte sort de la guerre avec la conviction que le partage de l'expérience traumatique et sublime de la guerre a offert à sa génération, la génération de ceux qui ont " compris la guerre ${ }^{41}$ », les moyens de créer une nouvelle société. Dans sa biographie politique, Giuseppe Pardini dit que réussir à comprendre ce qu'ont signifié pour Malaparte ces années de guerre équivaudrait à comprendre Malaparte lui-même ${ }^{42}$. Le secret de l'expérience restera intact jusqu'au bout, mais l'impact du "fait guerre " a irrigué l'œuvre entière comme le choc d'une balle fissure le verre en multiples éclats. Par-delà ses masques, ses postures et ses incarnations, l'engagement de l'écrivain-combattant a pris à chaque fois son impulsion d'un unique socle: la conscience d'appartenir à une génération et à une période historique, la conviction d'avoir reçu en héritage les complexités et les hypocrisies d'une Europe décadente, "pourrie», et la conscience de sa propre responsabilité 
d'homme et d'artiste : il s'agira, dès lors, de dénoncer cette " décadence » c'est-à-dire placer ses contemporains devant le miroir de leur propre aliénation, de leur « esclavage ».

Livre d'un survivant, Viva Caporetto! marque très clairement le passage à une écriture d'un autre type, une entrée en littérature, où l'on reconnaît déjà la nécessité pour l'écrivain d'écrire avec les mots des deux mondes : le monde d'ici, actuel et historique, et un monde souterrain, où l'expérience de la guerre l'a jeté, mythiquement lié à la terre, à l'histoire des hommes, au royaume des morts, psychiquement relié à un moi tour à tour blessé, anéanti et rené de la guerre. De nombreuses pages en porteront la trace, le souffle plutôt, qu'il appartient aux lecteurs de ressentir à leur tour pour lire, pour pouvoir lire, l'œuvre rebelle d'un écrivain qui a si bien compris son temps... à contretemps. Qui sait si un jour le souffle fabuleux de Viva Caporetto! n'abordera pas à des temps qui jugeront utile de réhabiliter et de réintégrer au sein d'une histoire nationale et européenne les prétendus déserteurs de Caporetto?

\section{NOTES}

1. C. Malaparte, Viva Caporetto! La rivolta dei santi maledetti, M. Isnenghi (éd.), Milan, Mondadori, 1980 ; C. Malaparte, Viva Caporetto! La rivolta dei santi maledetti, M. Biondi (éd.) Florence, Vallecchi, 1995 ; C. Malaparte, La rivolta dei santi maledetti, dans L'Europa vivente e altri saggi politici (1921-1931), E. Falqui (éd.), Florence, Vallecchi, 1961 ; C. Malaparte, La rivolta dei santi maledetti, dans Opere scelte, L. Martellini (éd.), Milan, Mondadori, « i Meridiani », 1997.

2. M. Biondi, Polemos. Le guerre di Malaparte, in Scrittori e miti totalitari, Malaparte, Pratolini, Silone, Florence, Edizioni Polistampa, 2002, p. 12-56.

3. Giuseppe Pardini dresse dans l'introduction de sa "biographie politique», en quelques citations bien choisies, un portrait aussi divertissant qu'affligeant, du Malaparte de légende, bien convaincu que l'autorité de cette "vulgate», bâtie autour de l'idée d'un "transformisme funambule, ambivalent, et incertain » (Isnenghi), est suffisamment puissante pour faire douter de la réussite de toute tentative de réhabilitation de l'auteur (G. Pardini, Curzio Malaparte. Biografia politica, Milan-Trente, Luni Editrice, 1998, p. 14-18). Pardini écrivait il y a près de 20 ans, les choses ont-elles changé aujourd'hui?

4. Dans cet article, nous retenons le nom choisi par l'écrivain en 1925, certes anachronique pour parler du jeune Suckert des années de guerre et d'après-guerre, mais qui réintègre a posteriori Viva Caporetto! dans la totalité de l'œuvre.

5. Pour une analyse de la première page de Viva caporetto! dans sa résonance avec la pensée politique et philosophique dont elle s'inspire, en particulier celle du philosophe transcendandaliste Ralph Waldo Emerson, cf. E. Mattiato, «Viva Caporetto! et le mythe malapartien des origines: résonances de Marinetti, Gobetti et Emerson (1921-1923)» dans le présent ouvrage. Sur l'héritage mazzinien du premier Malaparte, cf. également E. Mattiato, "Messianismo politico e antimodernità in Malaparte », Revue des études italiennes, n 1-2, janvierjuin 2009, p. 109-121.

6. Luigi Martellini évoque l'hypothèse de l'existence de ce rapport dans sa postface à La Rivolta dei santi maledetti, dans C. Malaparte, Opere, ouvr. cité, p. 1498-1499.

7. M. Biondi, Scrittori e miti totalitari, ouvr. cité, p. 99. 
8. L'expression est dans Viva Caporetto!, M. Biondi (éd.), ouvr. cité, p. 67, 69, 77. Malaparte écrivit pour les soldats dès le début de la guerre ; tous les écrits de la Grande Guerre ont été réunis par la sœur de Malaparte : E. Ronchi Suckert, Malaparte, I, Città di Castello, Ponte alle Grazie, 1991.

9. Viva Caporetto!, M. Biondi (éd.), ouvr. cité, p. 49.

10. Pour une chronologie détaillée de ces années, voir S. Laporte, Chronologie. Malaparte dans les années de la guerre et de l'après-guerre, dans C. Malaparte, Viva Caporetto!, traduction, préface et notes par Stéphanie Laporte, Paris, Les Belles Lettres, " Mémoires de guerre », 2012, p. 19-26.

11. Lettre à Giovanni Ansaldo du 8 août 1923, citée par L. Martellini, La Rivolta, ouvr. cité, p. 1501.

12. Depuis les travaux pionniers de E. J. Leed (No Man's Land. Combat \& Identity in World War I, Cambridge, Cambridge University Press, 1979), la littérature sur les «fous » de la Grande Guerre est aujourd'hui très abondante. Par exemple, parmi les plus récents, S. Tison et H. Guillemain, Du front à l'asile : 1914-1918, Paris, Alma Éditeur, 2013 et L. Tatu et J. Bogousslavsky, La folie au front. La grande bataille des névroses de guerre (1914-1918), Paris, Imago, 2012. Sur le sujet du traumatisme de guerre, nous renvoyons à l'excellent documentaire de Laurent Bécue-Renard, of Men and War (2014), qui filme le traitement du traumatisme et recueille le témoignage de douze soldats américains de retour d'Irak.

13. C. Rebora, Tra melma e sangue. Lettere e poesie di guerra, V. Rossi (éd.), Novare, Interlinea, 2008. C. Rebora, Chœur bouche close. Poèmes de guerre, 1914-1917, traduction et préface de S. Laporte et de P.-A. Claudel, Nîmes, Lucie éditions, 2009. Fortuitement parues au même moment, les deux publications relèvent de choix éditoriaux différents. Plusieurs proses de guerre figurent également dans la récente parution, dans la collection «I Meridiani », des écrits de Rebora avant sa conversion au catholicisme : C. Rebora, Poesie, prose, traduzioni, A. Dei (éd.), Milan, Mondadori, 2015.

14. G. B. Guerri, L'arcitaliano. Vita di Curzio Malaparte, Milan, Bompiani, 2008 (1980), p. 40.

15. C. E. Gadda, Giornale di guerra e di prigionia, Turin, Einaudi, 1999 (1965). Même en France ce texte (Journal de guerre et de captivité, Monique Baccelli (trad.), Paris, C. Bourgois, 1993) a été connu et diffusé avant celui d'Emilio Lussu (traduit sous le titre Les hommes contre, E. Genevois et J. Montfort (trad.), Paris, Austral, 1995).

16. Giovanna Procacci, Soldati e prigionieri italiani nella Grande Guerra, Rome, Editori Riuniti, 1993.

17. «É un testo che inneggia non alla guerra, eppure amata soprattutto quando la si odia, ma all'indimenticabile deposito umano che la vita in trincea ha lasciato nelle ossa e nel sangue del reduce, il senso profondo di una partecipazione collettiva, di una mistica umanissima, la nostalgia di un insieme che l'egoistica pace disgrega. » (M. Biondi, Polemos. Le guerre di Malaparte, dans Scrittori e miti totalitari, ouvr. cité, p. 52.)

18. Au moment d'observer la profonde mutation du Malaparte de Viva Caporetto!, Maurizio Serra propose deux dates, Caporetto et Bligny : « Mais comment expliquer la transformation intime qui d'un collégien prometteur, encore empâté dans ses tentatives d'éloquence scolaire, donnera un écrivain déjà mûr et formé, maître de ses qualités et de ses défauts, doué de l'originalité de ton qu'il gardera tout au long de son œuvre. À défaut d'explication suffisante, bornons-nous à signaler le poids de deux événements qui auront pour lui une valeur décisive : la rupture du front italien, en octobre 1917, et le nouveau départ pour la France, au printemps de l'année suivante. » (M. Serra, Malaparte. Vies et légendes, Paris, Grasset, 2011, p. 72)

19. C. Malaparte, Mamma marcia, Florence, Vallecchi, 1959, p. 125-134.

20. C. Malaparte, Viva Caporetto!, M. Biondi (éd.), ouvr. cité, p. 71.

21. Ibid., p. 95.

22. C. Malaparte, Ritratto delle cose d'Italia, degli eroi, del popolo, degli avvenimenti, delle esperienze e inquietudini della nostra generazione (prefazione alla seconda edizione romana del 1923), dans Viva Caporetto!, M. Biondi (éd.), ouvr. cité, p. 202-203. Il est aussi question de «maladie » (ibid., p. 187), d'« inquiétude » et de « fatigue » de l'après-guerre (ibid., p. 188).

23. C. Malaparte, Memoriale, dans Malaparte, I, ouvr. cité, p. 280-282. 
24. Il fut successivement, en mars 1923, responsable du Bureau de propagande internationale, puis en mai 1923 secrétaire général des Syndicats fascistes à l'étranger (cf. G. Pardini, Biografia politica, ouvr. cité, p. 85).

25. C. Malaparte, « Lettera à Binazzi, 11 avril 1921 », dans Malaparte, I, ouvr. cité, p. 203.

26. C. Malaparte, L'Europa vivente, dans Id., L'Europa vivente e altri saggi politici (1921-1931), ouvr. cité, p. 452.

27. C. Malaparte, Viva Caporetto!, M. Biondi (éd.), ouvr. cité, p. 82-83.

28. L'histoire littéraire de ces années de guerre ne s'abstrait jamais du politique. Cela est aisément admis pour les futuristes, moins pour les autres poètes de ces années-là. Cette hybridation du poétique et du politique agit avec une souplesse et une complexité d'autant plus difficiles à saisir que le poids (conscient ou inconscient) des complexes patriotiques et nationaux n'a pas relâché son emprise, même avec la fin du fascisme. La plus célèbre revue d'avant-garde poétique, Lacerba, ne s'est-elle pas muée en revue strictement politique, du jour au lendemain, pour défendre la nécessité de l'intervention de l'Italie aux côtés de la France ? Se souvient-on qu'au lendemain de l'intervention de l'Italie en mai 1915, une autre très célèbre revue culturelle, La Voce, a cessé toute publication politique et philosophique pour ne plus se consacrer qu'à une écriture poétique, entendue comme écriture apolitique, anticipant ainsi sur la prosa d'arte de la période fasciste? De même, les textes de Renato Serra, Piero Jahier, Giovanni Boine sont à la frontière des genres politique, historique et littéraire et sont mal connus aujourd'hui justement en raison de cette hybridation qui en fait toute la richesse. Cf. S. Laporte, Le fragmentisme italien (1912-1919) : brève histoire d'un genre littéraire, dans M. Orsino (éd.), La poésie italienne et la "Grande Guerre ", Collection de l'E.C.R.I.T., nº 8, Université de Toulouse-Le Mirail, 2004.

29. G. D'Annunzio, Notturno, Milan, Mondadori, 1991, p. 240-241.

30. Cf. S. Laporte, L'Océanisme: une passion cosmopolite du jeune Malaparte à la veille de son adhésion au fascisme (1921-1922), dans U. Lemke, M. Lucarelli et E. Mattiato (éds), Cosmopolitisme et réaction : le triangle France-Allemagne-Italie dans l'entre-deux-guerres, Chambéry, Éditions de l'Université Savoie Mont Blanc, Laboratoire LLSETI, 2014.

31. La formule est tirée de Oceanica, $\mathrm{n}^{\circ} 1,1^{\mathrm{er}}$ janvier 1921. Cf. aussi dans le numéro suivant: «Ritornare ai primitivi, alla loro semplicità naturale e capace di tutto comprendere, senza preconcetti e senza restrizioni, crearsi una morale indipendente dalle necessità della meschina vita d'ogni giorno ma aperta alla comprensione universale del tutto, ciò significa essere oceanici. » ("Che cos'è l'Oceanismo », Oceanica, 15 janvier 1921)

32. Chiffons, haillons et loques des soldats rendus visibles à chaque page de Viva Caporetto! et qui contrastent fortement avec les photographies de Malaparte en habit militaire impeccablement propre, boutonné et repassé.

33. Malaparte, I, ouvr. cité, p. 282.

34. C. Malaparte, Ritratto delle cose d'Italia..., ouvr. cité, p. 181-183.

35. Maurizio Serra fait la part belle dans sa biographie à la vie de combats que mena Malaparte, en insistant sur la force d'un écrivain " ascète » et " guerrier ».

36. C. Malaparte: «Nous disons aux étrangers : Que sous les pierres de nos monuments, qu'ils flairent avec tant de volupté malsaine, ne gît pas le cadavre de l'Italie et qu'il n'est pas encore temps de descendre chez nous en corbeaux; [...] Que les étrangers n'oublient jamais, même si l'Océanisme allait être de suite imité à Paris ou bien à Tipparery, que la première parole en est partie de l'Italie et que l'Océanisme reste et restera un mouvement international d'origine italienne. » (Oceanica, $1^{\mathrm{er}}$ janvier 1921 ; l'article est en français.)

37. « Nato in una città operaia, Prato, cresciuto ed educato in una famiglia dove gli operai di mio padre erano di casa (mio padre era un tecnico, nato dal popolo, non un industriale), io sentivo con particolare intensità i problemi del lavoro. I problemi della classe operaia non mi erano, non mi sono, non mi saranno mai estranei. A ciò debbo questa mia fedeltà agli operai, questa mia predilezione un po' letteraria, forse, certo molto intellettualistica, in un certo senso, per i 
problemi della vita operaia cui non sono mai venuto meno in quasi trent'anni di attività di scrittore. Parlai e scrissi a Piero Gobetti della situazione in cui erano venuti a trovarsi gli operai italiani, dopo il fallimento dello "sciopero legalitario" dell'Agosto 1922, gli proposi di lanciar l'idea di una organizzazione sindacale, basata sull'accettazione di alcuni postulati "nazionali". Gobetti mi rispose che per la classe operaia italiana non v'era altra salvezza che il marxismo integrale, e rigettò la mia proposta. In quanto alla mia idea di cui avevamo discusso tante volte insieme, che fosse molto più utile tentar di modificare il fascismo dal di dentro che combatterlo dal di fuori, egli mi rispose che tentar di modificare il fascismo dall'interno del P.N.F. sarebbe stata fatica sprecata, e che bisognava invece combatterlo con tutte le forze e con la più assoluta intransigenza. » (Malaparte, I, ouvr. cité, p. 282.)

38. C. Malaparte, Ritratto delle cose d'Italia, ouvr. cité, p.179. Les expressions de ce conflit intimement malapartien sont innombrables; Pardini cite par exemple, un intéressant article où Malaparte imagine un combat entre son moi littéraire et son moi politique : C. Malaparte, «Un duello mortale », L'Italia letteraria, juillet 1927 (cf. G. Pardini, Biografia politica, ouvr. cité, p. 211).

39. Un groupe d'amis artistes, « quelques hommes sortis du tourment [de la guerre] comme des entrailles d'une seconde mère [...] seront des réservoirs d'infini » («Lettera ad un Oceanico svizzero », Oceanica, n 3, 15 février 1921, p. 1 [l'article est en français]).

40. "Non è un libro di guerra, questo ", "Questa non è una raccolta d'impressioni di guerra, né un diario » (C. Malaparte, Viva Caporetto!, M. Biondi (éd.), ouvr. cité, p. 48 et 76).

41. Les occurrences du verbe capire, souvent employé sans objet, sont très nombreuses et rendent compte de la nature particulière du texte: un rapport sur le cheminement qui a porté à la compréhension de la guerre et non sur la guerre elle-même.

42. "Capire le motivazioni, le ragioni, i risultati di quella sua esperienza vuol dire anche, per molti aspetti, comprendere Malaparte » (G. Pardini, Biografia politica, ouvr. cité, p. 35). Pardini fait de Viva Caporetto! le fondement de l'œuvre et décrit très bien comment se construit à partir de la guerre la ligne idéologique de l'auteur. Une ligne agrégée de façon cohérente, même si les frontières en sont souples, autour de la notion de « révolution conservatrice ».

\section{RÉSUMÉS}

De retour de cinq années de guerre, Curzio Malaparte a 22 ans lorsqu'il écrit et publie Viva Caporetto!, à la fois témoignage individuel de premier plan et interprétation politique de la Grande Guerre. Contre la propagande officielle, il choisit pour emblème de l'héroïsme du soldat des tranchées et espoir de revanche d'un peuple méprisé, Caporetto, la gigantesque retraite des troupes italiennes sous l'avancée des armées autrichiennes qui marqua, en octobre 1917, la crise militaire la plus douloureuse que l'Italie ait connue. Trois fois saisi et censuré entre 1921 et 1923, Viva Caporetto! était une charge explosive contre la jeune Italie fasciste qui s'édifiait sur la mémoire d'une Grande Guerre victorieuse. Ce récit de guerre hors du commun marque, en même temps que l'entrée en littérature du jeune Malaparte, la matrice de toute son œuvre.

Dopo cinque anni di guerra, soldato sui fronti italiano e francese, Curzio Malaparte ha 22 anni quando scrive e pubblica Viva Caporetto!, insieme testimonianza individuale e interpretazione politica della Grande Guerra. Contro la propaganda ufficiale, sceglie come emblema dell'eroismo dei soldati e simbolo di rivincita Caporetto, gigantesca ritirata delle truppe italiane davanti agli eserciti austriaci, che segna, nel mese di ottobre 1917, la crisi militare più dolorosa che l'Italia 
abbia conosciuto. Tre volte censurato tra il 1921 e il 1923, Viva Caporetto! fu una carica esplosiva contro la giovane Italia fascista che si era costruita sul mito di una Grande Guerra vittoriosa. Questo racconto di guerra fuori del comune, rappresenta insieme l'ingresso del giovane Malaparte nel mondo delle lettere e la matrice di tutta l'opera futura.

Returning from 5 years of war, Curzio Malaparte is 22 years old when he writes and publishes Viva Caporetto!, which is both an individual testimony and a political interpretation of the Great War. Against the official propaganda, Malaparte turns Caporetto-the gigantic retreat of the Italian troops following the advance of the Austrian army in October 1917-into an emblem of the heroism of the soldiers that were in the trenches and of their hope for revenge. The event stroke Italy as the worst military crisis the country ever knew. Seized and censored three times between 1921 and 1923, Viva Caporetto! undermined the myth of a victorious and glorious Great War on which young Fascist Italy built its rhetoric. This unique war story was both Malaparte's literary debut and the source of his entire work.

\section{INDEX}

Mots-clés : Curzio Malaparte, Viva Caporetto!, La Rivolta dei santi maledetti, Première Guerre mondiale, déroute de Caporetto, littérature de tranchées, anciens combattants, syndrome de stress post-traumatique

Parole chiave : Curzio Malaparte, Viva Caporetto!, La Rivolta dei santi maledetti, Prima guerra mondiale, rotta di Caporetto, letteratura di trincee, reducismo, disturbo post-traumatico da stress

Keywords : Curzio Malaparte, Viva Caporetto!, La Rivolta dei Santi Maledetti, First World War, Battle of Caporetto, trench literature, Veterans, Post-traumatic stress disorder

\section{AUTEUR}

\section{STÉPHANIE LAPORTE}

Lycée international de Saint-Germain-en-Laye, Sciences-Po Saint-Germain-en-Laye 